\title{
Neuroimaging in Dementia
}

\author{
Meike W. Vernooij and Mark A. van Buchem
}

\section{Learning Objectives}

- Understanding that dementia is a clinical syndrome with a variety of underlying pathologies or subtypes (varying by age of presentation of the patient); and that neuroimaging plays an important role in guiding clinical diagnostic assessment.

- Gaining insight into the distinction between imaging findings in "normal aging" versus those in neurodegeneration.

- Being able to list the most typical imaging findings of the most common subtypes of dementia.

- Acquiring knowledge on the recommended imaging protocol in the memory clinic setting as well as the elements essential for the radiological report.

\subsection{Dementia: A Syndrome, Not a Disease}

Dementia is an acquired syndrome in which a person suffers from progressive impairment in one of the following six cognitive domains: complex attention, executive function, learning and memory, language, perceptual-motor function, and/ or social cognition. These cognitive deficits cannot be attributed to another mental disorder (e.g., depression or schizophrenia) and must be severe enough to interfere with independent daily functioning to be classified as a "major neurocognitive disorder" (major NCD) according to the

M. W. Vernooij $(\bowtie)$

Department of Radiology and Nuclear Medicine, Erasmus MC University Medical Center, Rotterdam, The Netherlands

Department of Epidemiology, Erasmus MC University Medical Center, Rotterdam, The Netherlands

e-mail: m.vernooij@erasmusmc.nl

M. A. van Buchem

Department of Radiology, Leiden University Medical Centre,

Leiden, The Netherlands

e-mail: m.a.van_buchem@lumc.nl
DSM-V 2013 edition [1]. This new choice in terminology in the DSM-V was chosen in order to be able to also define a "mild neurocognitive disorder" (equivalent to mild cognitive impairment (MCI) and/or prodromal dementia), with the key distinction that persons with mild NCD remain independent in daily functioning. This shift to diagnose NCD as early as possible stems from the understanding that these diseases have a long prodromal phase, in which early diagnosis and early treatment may have beneficial effects. Importantly, mild NCD is not always a precursor to major NCD (dementia), and the diagnosis does not require further decline.

The syndrome of dementia has a multitude of underlying causes and the diagnostic work-up is primarily aimed at identifying these and to assign a "subtype." The clinical work-up relies heavily on history taking, clinical and neuropsychological (cognitive) assessment and can be complemented by a number of ancillary tests, of which neuroimaging is one of the most important.

\subsection{Dementia: Population Prevalence and Subtype Proportions}

Due to the aging of populations worldwide, the absolute number of cases of dementia is estimated to double worldwide between 2020 and 2040, to a staggering 81 million over the age of 60 living with dementia in the year 2040, with the highest rise in developing countries [2]. Pooled prevalence data of 11 European population-based studies published in 2000 showed the following estimates of prevalence of dementia in the elderly ( $>65$ years): age-standardized prevalence was $6.4 \%$ for dementia (all causes), $4.4 \%$ for Alzheimer's disease (AD), and $1.6 \%$ for vascular dementia (VaD). Prevalence of dementia was higher in women than in men and nearly doubled with every 5 years increase of age: from $0.8 \%$ in the group age 65-69 years to $28.5 \%$ over the age of 90 years [3].

In the elderly, the most common underlying cause (or subtype) of dementia is Alzheimer's disease (45-54\%), followed by vascular dementia (14-16\%), Lewy body (5\%), 


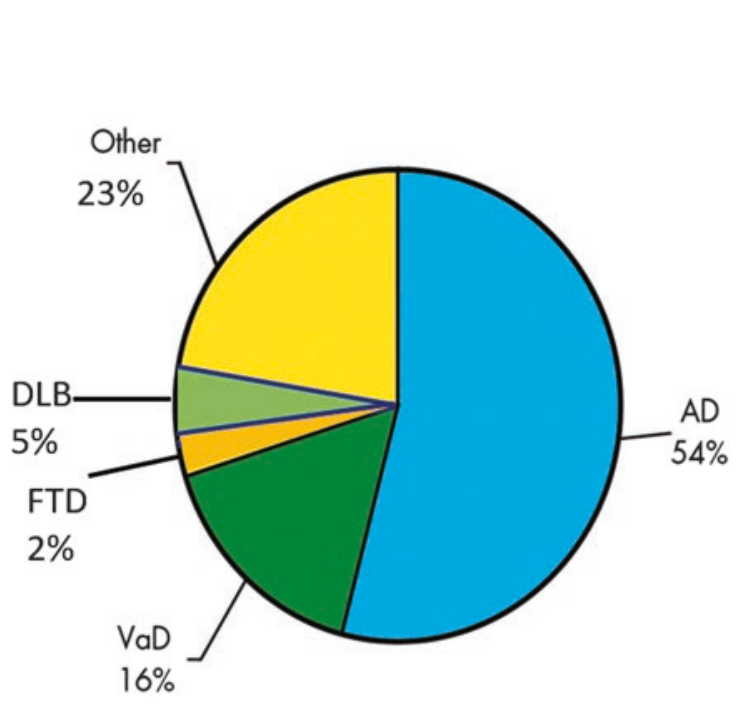

Age $\geq 65$ years

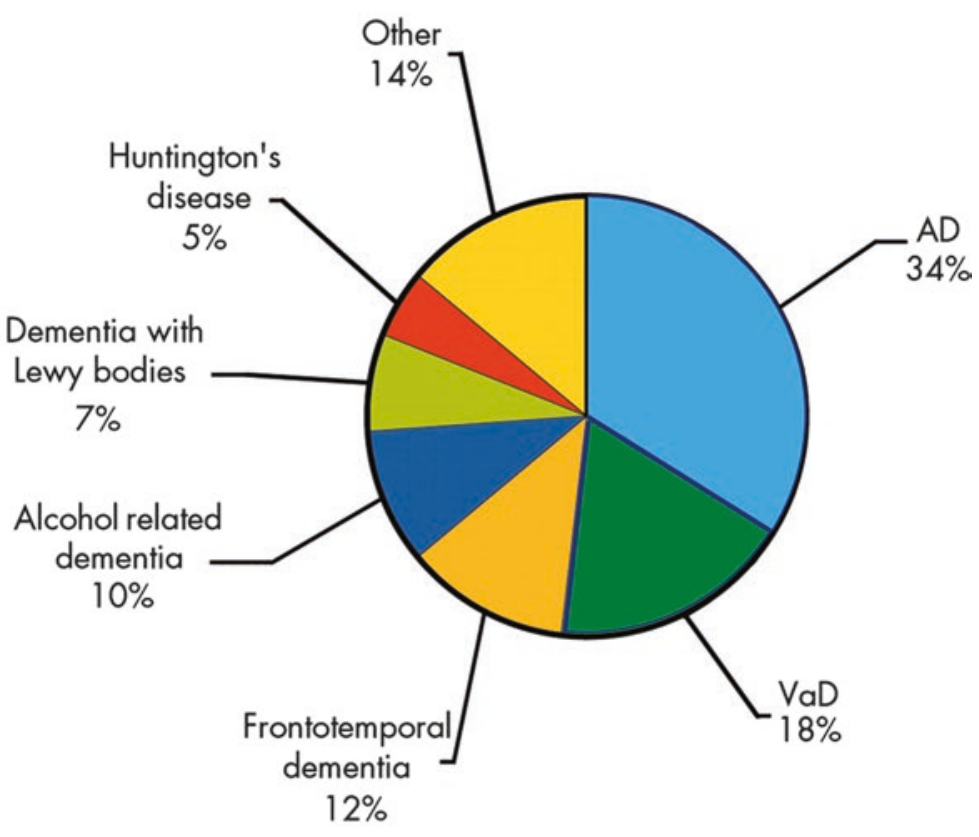

Age $<65$ years

Fig. 11.1 Subtype proportions of dementia according to age of onset. Adapted from van der Flier and Scheltens [5]

frontotemporal dementia (2\%), and alcohol induced dementia $(0.7 \%)[4,5]$ (Fig. 11.1).

It is important to realize that in early onset dementia ( $<65$ years), these subtype proportions differ, with the primary clinical subtypes still being Alzheimer's disease (18-34\%) and vascular dementia (13-18\%), but now with a much higher proportion of frontotemporal dementia (11-12\%) [6, 7]. Also other causes such as alcohol-related dementia (9-18\%), traumatic brain injury, Huntington's disease, and HIV-related dementia are more prevalent in early onset dementia versus late onset (Fig. 11.1). Due to the fact that younger patients ( $<65$ years) more often have alternative diagnoses or a genetic cause of dementia, imaging plays an even more important role in this group to aid clinical diagnosis, in particular since different subtypes bring about different prognostic consequences.

It is crucial to understand that in many cases, especially in the elderly, there are multiple causative factors, of which a combination of Alzheimer pathology and vascular pathology is one of the most prominent ("mixed pathology," not yet indicated in Fig. 11.1 but in late onset dementia responsible for up to $30-40 \%$ of dementias) [8]. This should be recognized in the diagnosis and should be kept in mind when reading imaging exams of memory clinic patients.

Furthermore, the proportion of subtypes differ according to autopsy versus clinical diagnosis [9] indicating that current clinical criteria for subtyping dementia may not adequately capture the true underlying pathology. This is also the reason why in the field of research, biomarkers have become an essential component which have also been incor- porated in new, unbiased descriptive classification schemes that do not specify disease labels. One of these is the "A/T/N" system [10], in which seven major AD biomarkers are used to define three binary categories for presence/absence of these markers (amyloid in PET or CSF ("A"); tau on PET or in CSF ("T") or neurodegeneration on FDG PET, MRI, or CSF total tau ("N")). Each biomarker category is rated as positive or negative. An individual score might, for example, appear as $\mathrm{A}+/ \mathrm{T}+/ \mathrm{N}-$, or $\mathrm{A}+/ \mathrm{T}-/ \mathrm{N}-$, etc. Given the present lack of consensus among $\mathrm{AD}$ specialists on terminology across the clinically normal to dementia spectrum, a biomarker classification scheme will have broadest acceptance if it is independent from any clinically defined diagnostic scheme. Though such a biomarker-based classification will not be implemented in clinical practice in the short term, it is important for radiologists to be aware of the shifting views in the field on traditional disease labels.

\section{Key Points}

- Dementia is a syndrome with a multitude of underlying causes, the proportions of these differ for early onset ( $<65$ years) versus late onset dementia.

- Alzheimer's disease and vascular dementia are at all ages the most common subtypes according to clinical diagnosis, but neuropathology studies show a very high proportion of mixed pathology (AD pathology + vascular pathology), up to $30-40 \%$ in old age. 


\subsection{Dementia: Role of Neuroimaging}

Neuroimaging is one of the most important ancillary tests in the clinical work-up of a patient suspected of dementia. The primary aim of imaging used to be the exclusion of treatable causes of cognitive impairment (such as subdural hematomas, brain tumors, or normal pressure hydrocephalus), but imaging is increasingly helpful in the identification of underlying causes of dementia. Though for the majority of diseases causing dementia, no specific imaging criteria have been formulated in the clinical diagnostic criteria, it is notable that more recent revisions of criteria are increasingly including imaging (for positive as well as negative predictive value) [11].

Though Alzheimer's disease is at any age the most prevalent dementia subtype, the most important differential diagnoses of $\mathrm{AD}$, depending on age of onset, are vascular dementia (VaD), FTD, non-fluent and semantic subtypes of primary progressive aphasia (PPA), dementia with Lewy bodies (DLB), and Creutzfeldt-Jakob disease (CJD). Especially in the early stages of the disease, when symptoms are often unspecific, differential diagnosis may be challenging. Accurate differentiation between the several types of dementia, however, is of especially great relevance in the early stages, when most can be gained in terms of symptom reduction, informing the patient on the prognosis and increased quality of life. Also, early and accurate differentiation is important for inclusion into clinical drug trials and therapy evaluation in a trial setting. Neuroimaging may play an important role in this regard.

\subsection{Dementia Versus Normal Aging}

This section contains text extracts that are reprinted with permission from Vernooij and Barkhof [12].

In the evaluation of brain imaging in a memory clinic setting, knowledge on the background of "normal" brain aging and its associated changes is important but often overlooked. Autopsy studies in brains of aged people without neurological disease invariably show the presence of plaques, tangles, Lewy bodies, and neuronal loss. These are pathological findings that are also considered hallmarks of neurodegenerative diseases, but are more abundantly present and regionally clustered in those diseases. This observation that almost all aged brains show characteristic changes that are strongly linked to neurodegeneration has led to the hypothesis that normal brain aging and neurodegenerative diseases form a continuum, and that pathological neurodegeneration perhaps is an expression of accelerated aging. Though this is likely a too simplified view, it is a fact that there is considerable overlap between patterns of brain pathology in aging and neurodegenerative disease and that knowledge on changes in brain aging is indispensable as a background against which to evaluate neurodegenerative disease.

The most important imaging findings in aging which overlap with those found in neurodegenerative diseases are brain atrophy and imaging markers of small vessel disease. Below we describe shortly those findings in the age-related setting, and when these can still be considered normal for aging or when they can provide an explanation for dementia.

\subsubsection{Brain Atrophy in Aging}

Regarding brain atrophy, there is consistent evidence that brain volume declines with aging, and that age-related volume loss appears as early as the age of $30(0.2 \%$ loss of total brain volume per year), and accelerates after the age of 70 up to $0.5 \%$ total brain volume loss per year (5\% per decade) in cognitively healthy elderly [13]. At the age of 75 , this roughly equates to a $10 \%$ brain volume loss, which is readily appreciable to the naked eye (Fig. 11.2).

The most important rules of thumb to distinguish atrophy in aging from neurodegeneration are that age-related brain atrophy is generally symmetric and generalized, whereas in neurodegeneration we see specific lobar patterns (further described below) and can see asymmetries. Semi-quantitative visual rating scales such as the global cortical atrophy scale (GCA) and medial temporal atrophy scale (MTA) (Tables 11.1 and 11.2, Figs. 11.3 and 11.4) are be helpful to distinguish "normal" from "abnormal" based on a visual assessment. Though these scales are quite crude and not sensitive to subtle or longitudinal changes, they have consistently been shown to correlate well with pathology data and with volumetric assessments and are easy to use in everyday clinical practice.

Yet, without absolute quantification it is very hard to assess what "normal" or even "expected" is for a specific age and sex, in particular when assessing atrophy on a regional or substructure level. Advances are being made in image analysis algorithms to perform fully automated tissue and structure quantification on individual level scans and compare these to normative databases, in order to aid everyday radiology assessments (Fig. 11.2).

On visual rating, asymmetry and focal brain atrophy should be considered abnormal, as is a GCA score of 3 ("knife-blading") in all persons or a score of 2 (clear gyral shrinking) in persons aged $<75$ years.

In individuals until age 75 years, the cutoff for abnormal MTA lies at an average of $\geq 1.5$, i.e., having a score of 2 (or higher) on one side is considered abnormal. In those over age 75 years, having an MTA score of 2 (or higher) on both sides (i.e., an average of $\geq 2.0$ ) is considered abnormal [17]. 


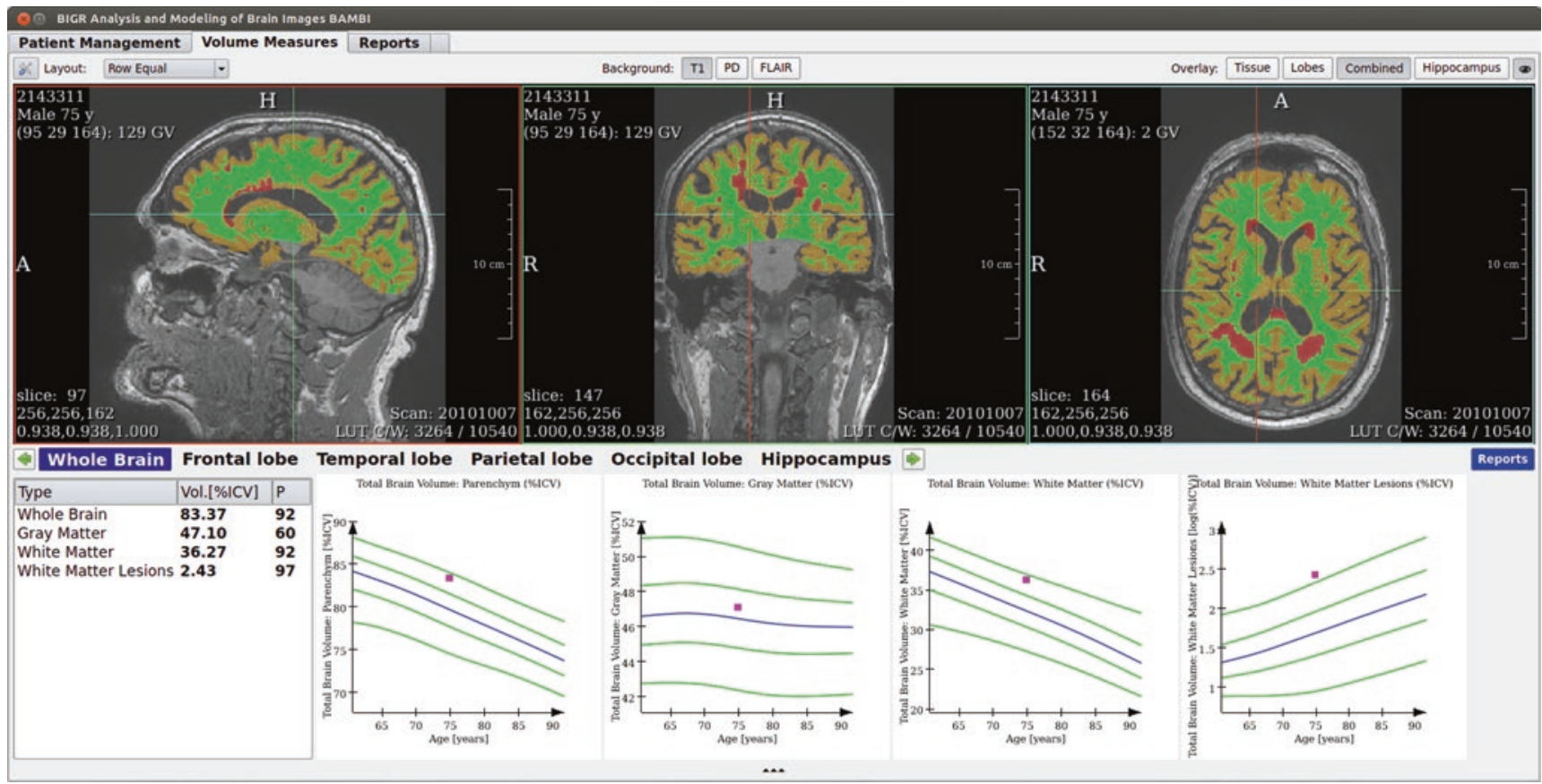

Fig. 11.2 Automated volumetry in the memory clinic. Screenshot example showing brain tissue volumetric results and application of reference curves to assess individual brain atrophy. (Reprinted with permission from Vernooij and Barkhof [12])

Table 11.1 Visual rating scale of global cortical atrophy (GCA)

\begin{tabular}{|c|c|c|c|}
\hline \multicolumn{2}{|c|}{ Degree of atrophy } & Gyri & Sulci \\
\hline $\mathrm{GCA}=0$ & No & Normal volume & $\begin{array}{l}\text { Normal } \\
\text { width }\end{array}$ \\
\hline $\mathrm{GCA}=1$ & $\begin{array}{l}\text { Mild (may be } \\
\text { considered normal in } \\
\text { the elderly) }\end{array}$ & Normal & $\begin{array}{l}\text { Some } \\
\text { opening of } \\
\text { sulci }\end{array}$ \\
\hline $\mathrm{GCA}=2$ & Moderate & Reduced & Enlarged \\
\hline $\mathrm{GCA}=3$ & Severe & $\begin{array}{l}\text { Severely reduced } \\
\text { ("knife blade") }\end{array}$ & $\begin{array}{l}\text { Severely } \\
\text { enlarged }\end{array}$ \\
\hline
\end{tabular}

Data from Pasquier et al. [14]
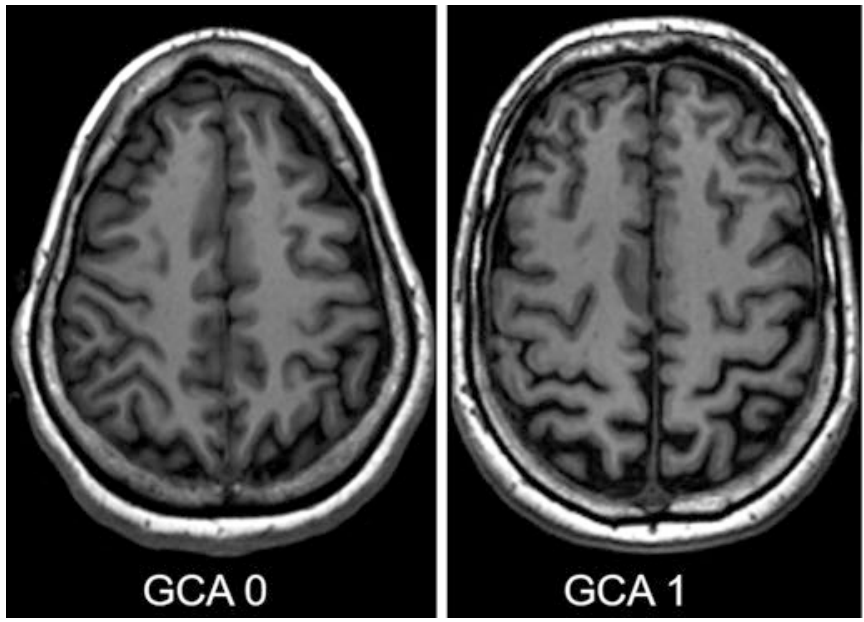

Fig. 11.3 Global cortical atrophy scale. Illustration showing the different categories on the GCA scale (see also Table 11.1): no atrophy in the parietal lobe (GCA 0), some opening of sulci (GCA 1), reduced volume
Table 11.2 Visual rating scale of medial temporal lobe atrophy (MTA)

\begin{tabular}{|l|l|l|l|} 
& Choroid fissure & Temporal horn & $\begin{array}{l}\text { Hippocampal } \\
\text { height }\end{array}$ \\
\hline MTA $=0$ & Normal & Normal & Normal \\
\hline MTA $=1$ & Widened & Normal & $\begin{array}{l}\text { Normal } \\
\text { Reduced }\end{array}$ \\
\hline MTA $=3$ & $\begin{array}{l}\text { Soderately } \\
\text { widened }\end{array}$ & Widened & $\begin{array}{l}\text { Moderately } \\
\text { reduced }\end{array}$ \\
\hline
\end{tabular}

MTA = 4 Severely widened Severely widened Severely reduced Data from Scheltens et al. [15]
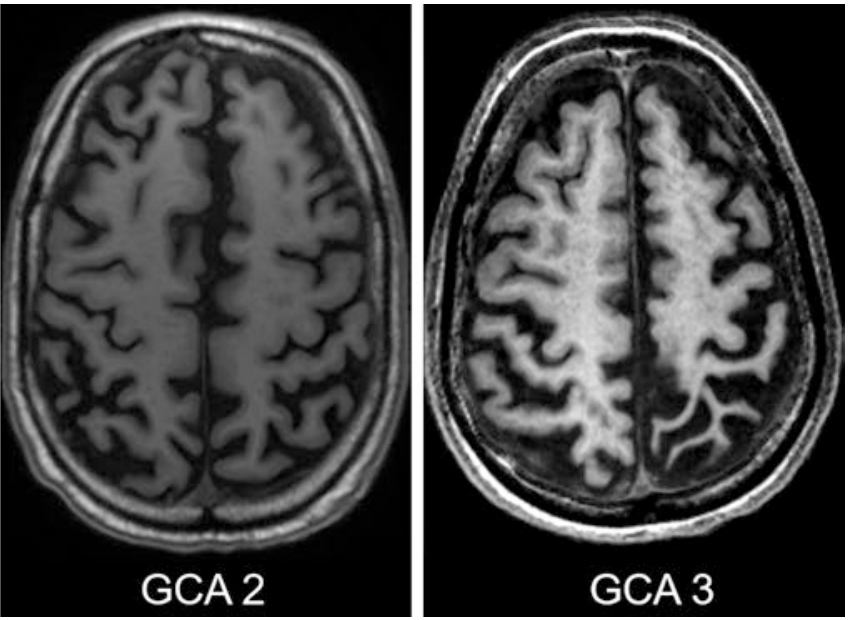

of gyri and further widening of sulci (GCA 2), and severe volume reduction (knife-blading) in the left parietal lobe (GCA 3) 


\subsubsection{Vascular pathology in aging}

Vascular pathology commonly seen in aging and which may overlap with vascular pathology in mild and major NCD, are white matter hyperintensities $(\mathrm{MH})$, silent brain infarcts (usually lacunes), and cerebral microbleeds.

Studies in community-dwelling subjects have shown that after the age of 45 years, only $5-10 \%$ of persons are completely free of $\mathrm{WMH}$, and that their presence and volume increase steeply with age. Large observational studies have provided compelling evidence of links of WMH with risk of stroke, dementia, cognitive dysfunction (in particular executive function and information processing speed), gait disturbance, and falls as well as depression. All of these observations have led to WMH being considered an expression of cerebral small vessel disease and not an innocuous finding, especially when larger in volume. In everyday clinical practice, standardized visual rating scales such as the Fazekas scale as described in Fig. 11.5 can be applied. Due to ease of use, this scale shows very high intra- and interrater reliability. In general, a Fazekas score of 1 can be con- sidered normal, whereas scores 2 and 3 indicate the presence of small vessel disease. A score of 3 is abnormal at any age, and found both in frontal and parietal lobes, is indicative of a large $\%(>25 \%)$ of white matter being pathologic and contributes to the clinical criteria for vascular dementia.

Silent brain infarctions are highly frequent in asymptomatic aging individuals, with prevalence estimates ranging from $8 \%$ to as high as $28 \%$ and increase with age [18]. Most of these infarcts are lacunes in the basal ganglia or deep white matter, presumed to be caused by underlying hypertensive small-vessel disease or small emboli. Although by definition silent infarcts go without overt clinical symptoms, there is compelling evidence that they are associated with subtle deficits in physical and cognitive function [18]. Moreover, the presence of silent infarcts, both lacunes and microinfarcts, more than doubles the risk of stroke and dementia, especially when multiple lesions are present.

Microbleeds, visible as hypointense focal lesions on T2*weighted sequences susceptible to the paramagnetic properties of blood-break down products, are thought to represent small chronic hemorrhages caused by damaged small vessels.
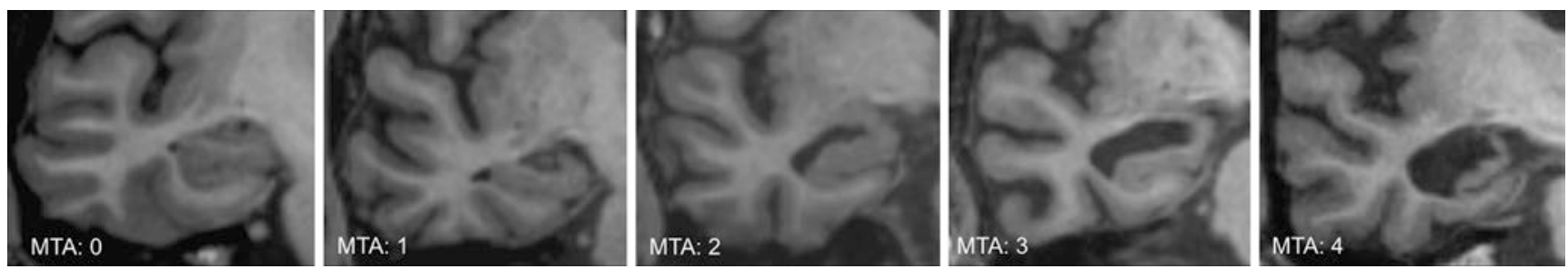

Fig. 11.4 Medial temporal atrophy scale. Coronal T1-weighted images of the hippocampus for stages $0-4$ of the MTA rating scale (see Table 11.2). Note progressive widening of the choroid fissure (from stage 1 onwards) and temporal horn (from stage 2 onwards), as well as hippocampal body volume loss (stages 3 and 4). (Reprinted with permission from Vernooij and Smits [16]. Copyright Elsevier (2012))
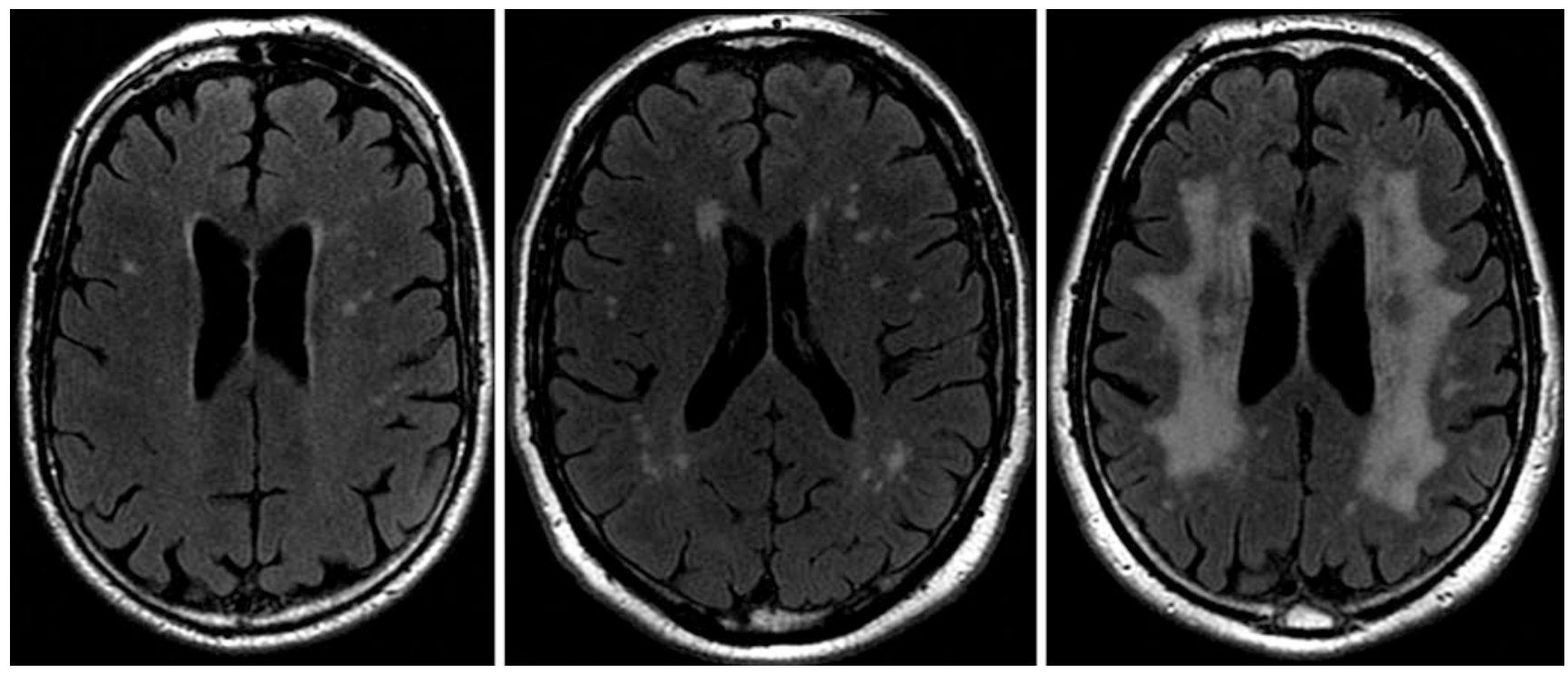

Fig. 11.5 Fazekas scale for white matter lesions. From left to right, Fazekas scale 1 (punctiform lesions), 2 (early confluent lesions), and 3 (confluent lesions) (not shown: score $0=$ no lesions). (Reprinted with permission from Vernooij and Smits [16]. Copyright Elsevier (2012)) 
Population cohorts in healthy elderly describe prevalence estimates ranging from 5 to $30 \%$, with increasing prevalence with age, but also with magnetic field strength, hindering easy comparison between populations or pooling of results. The topographic distribution of microbleeds is thought to reflect the type of underlying vasculopathy. Lobar microbleeds, especially those that are multiple and strictly lobar (cortico-subcortical junction of cerebral lobes) in location, are presumed to be caused by amyloid angiopathy, whereas deep microbleeds (basal ganglia, thalamus, brainstem, cerebellum) reflect hypertensive arteriopathy. Generally, a single microbleed in any brain location can be considered to be not of clinical relevance. However, having multiple (two or more) microbleeds in strictly lobar brain locations in a person aged over 55 years without other causes for microbleeds (e.g., previous radiotherapy, head trauma) classifies as "probable CAA" according to the Boston criteria. Due to the frequent co-occurrence of CAA and $\mathrm{AD}$ pathology, multiple lobar microbleeds can often be observed in patients fulfilling the clinical criteria of AD. The clinical diagnosis of CAA is further supported if superficial siderosis is also present (see Fig. 11.6). Similarly, having multiple microbleeds in deep brain locations or in the brainstem should trigger the presence of hypertensive arteriolosclerosis. Cerebellar microbleeds can occur both in the context of CAA or hypertension and thus are not contributing meaningfully to differential diagnosis.

\section{Key Points}

- The aging brain shows characteristic changes that are strongly linked to neurodegeneration.

- On visual rating, asymmetry and focal brain atrophy should be considered abnormal, as is a GCA score of 3 ("knife-blading") in all persons or a score of 2 (clear gyral shrinking) in persons aged $<75$ years.
- In individuals up to 75 years, an MTA score of 2 or higher on one side is abnormal, whereas in persons over the age of 75, a score of 2 or higher on both sides is considered abnormal.

- A Fazekas score of 2 or 3 is indicative of underlying small vessel disease.

- Silent brain infarcts, even though very frequent in aging subjects, more than double the risk of stroke and dementia and should be considered as indicative of small vessel disease.

- Microbleeds in lobar, deep, or cerebellar locations occur frequently in old age (up to $30 \%$ ). Generally, a single microbleed is considered not relevant, whereas the presence of multiple microbleeds raises suspicion of underlying cerebral amyloid angiopathy (when strictly lobar in location) or hypertensive arteriopathy (when deep or in brainstem).

\subsection{Imaging Characteristics of Most Common Subtypes of Dementia}

This section contains text extracts from Vernooij and Smits [16]. Copyright Elsevier (2012); reprinted with permission.

\subsubsection{Typical Imaging Presentation of Alzheimer's Disease}

The radiological hallmark finding of AD is cortical atrophy due to neuronal degeneration and loss. Atrophy is diffuse, but more prominent in the temporal and parietal lobes with the hippocampus most severely, and disproportionately
Fig. 11.6 Superficial siderosis and lobar microbleeds. T $2 *$ gradientecho images (or susceptibility-weighted images) showing linear hypointensity on the cortical surface (left), compatible with superficial siderosis, and multiple focal hypointensities in the brain parenchyma in a lobar location (right), reflecting cerebral microbleeds
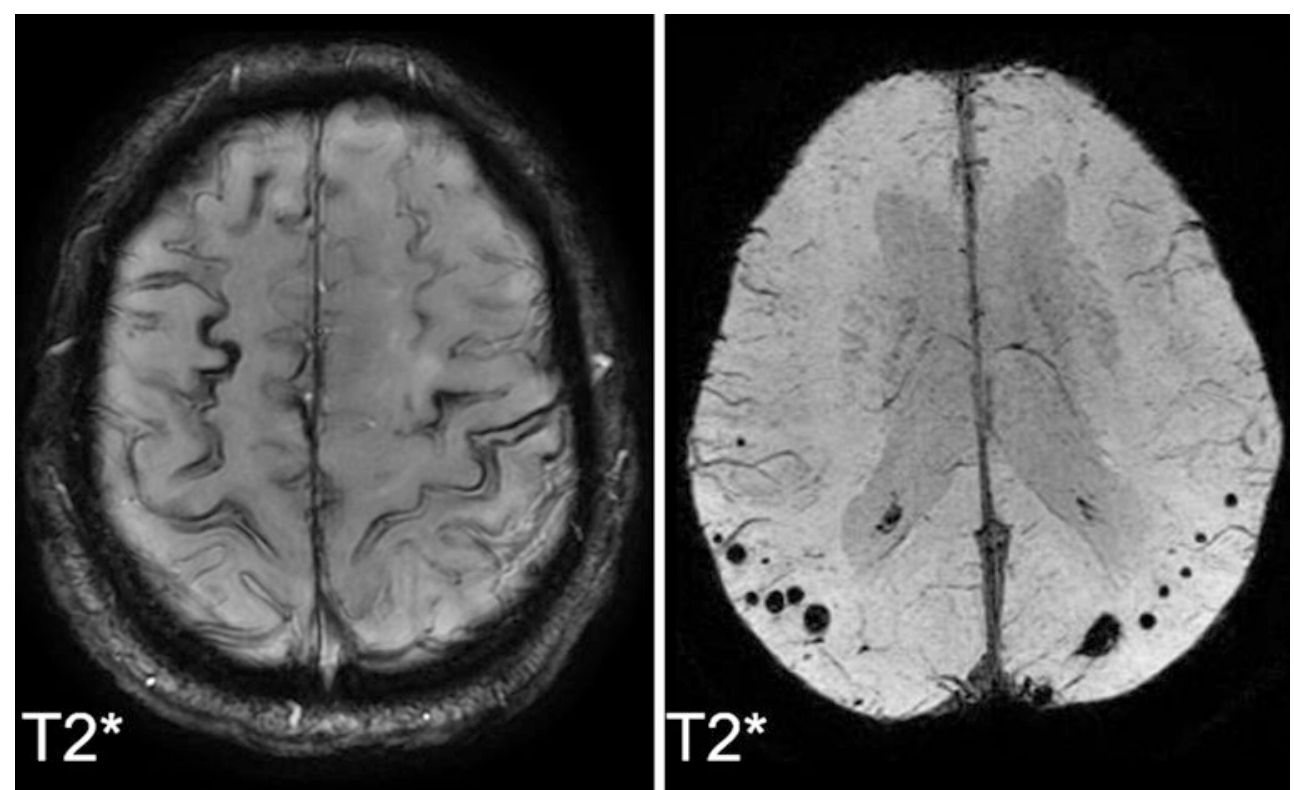
affected (Fig. 11.7). The primary motor and sensory cortices are relatively spared until late in the disease. Findings are bilateral and generally symmetrical, but a certain degree of asymmetry may occur.

Sensitivity and specificity to use MTA in distinguishing patients with $\mathrm{AD}$ from healthy controls are in the range of 85 and $88 \%$, respectively. Note that hippocampal atrophy is also common in other types of dementia, such as dementia with Lewy bodies, frontotemporal dementia, and the Heidenhain variant of Creutzfeldt-Jakob disease. Consequently, diagnostic accuracy to distinguish AD from other dementia is considerably lower than from healthy controls.

Asymmetry of the hippocampus does not exclude AD, although marked asymmetry suggests alternative diagnosis, most notably frontotemporal dementia (FTD, see further).

Fig. 11.7 Typical atrophy pattern in AD. Seventy-yearold female with clinical dementia. Coronal T1-weighted images show bilateral hippocampal atrophy (MTA 3 both sides, panels 1 image shows bilateral symmetric cortical atrophy, most prominent in parietal lobes. This pattern is typical for atrophy in (late onset) Alzheimer's disease and 2) and axial T1-weighted
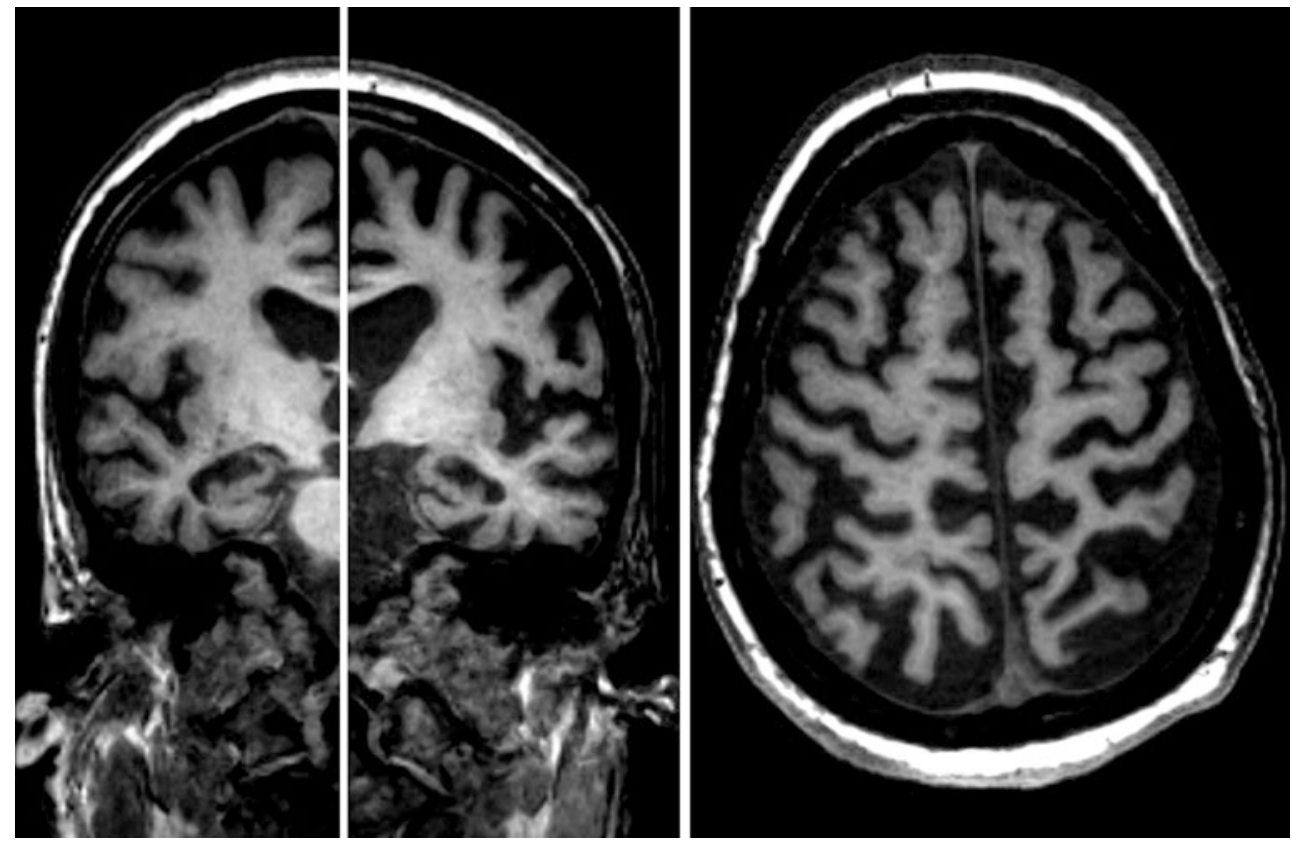

Fig. 11.8 Posterior cortical atrophy (precuneus atrophy) in atypical AD. Fifty-six-yearold female with clinical dementia. Sagittal T1-weighted image shows precuneus atrophy (and widening of the parietooccipital sulcus), whereas there is a normal hippocampal volume on coronal

T1-weighted image (MTA 1). This pattern is typical for posterior cortical atrophy in (early onset) Alzheimer's disease
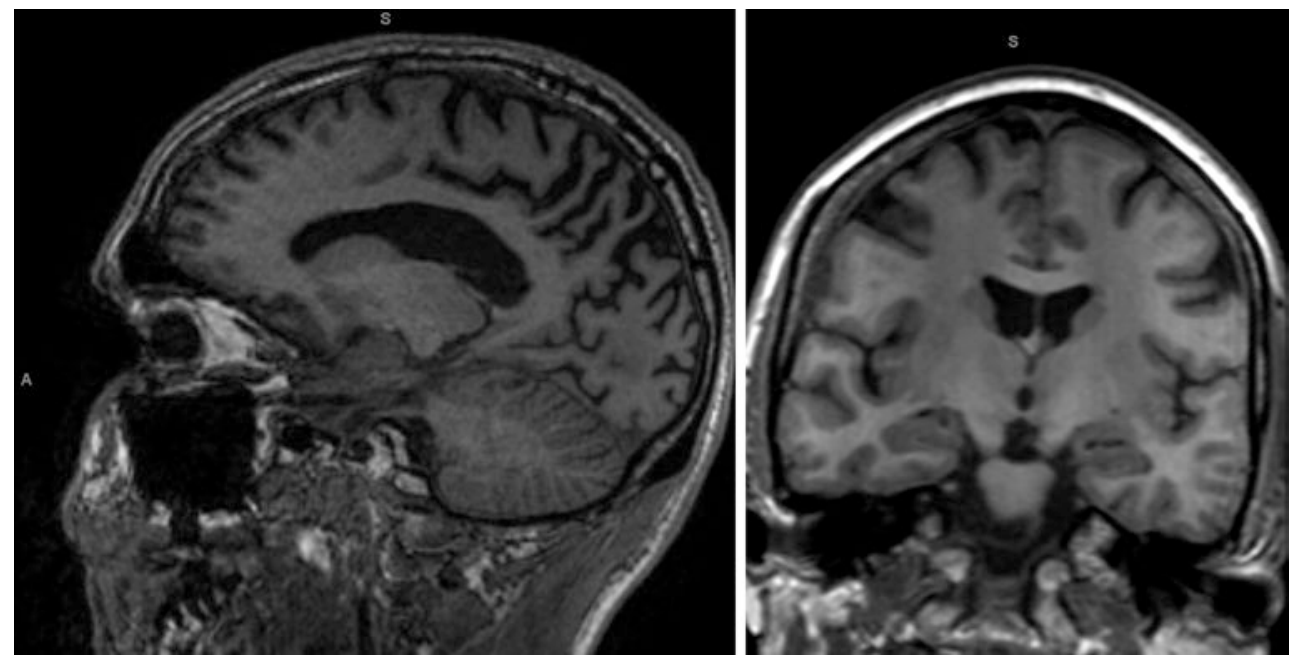
less prominent memory loss and show associated atrophy of the parieto-occipital and posterior temporal cortices. Atrophy is generally asymmetrical, the right hemisphere being more affected than the left. Though the underlying pathological substrate may differ, Alzheimer pathology was found to be most prevalent in these patients.

Logopenic progressive aphasia has been described as a clinically, neuropathologically, and radiologically distinct subtype of primary progressive aphasia (PPA, see more information in Sect. 11.5.4). The syndrome is characterized by language disorders rather than memory impairment, which consists of slow speech and deficits in sentence repetition. On brain imaging there is marked asymmetrical atrophy of the posterior temporal cortex, including the posterior superior and middle temporal gyri, and the inferior parietal lobule, with the left hemisphere more affected than right. Clinically, LPA is difficult to distinguish from the other PPA subtypes, which have predominantly underlying FTD pathology. Structural brain imaging plays an important role in differential diagnosis.

\subsubsection{Frontotemporal Dementia (FTD)}

FTD is in particular in patients under the age of 65 years a frequent occurring cause of dementia, occurring in up to $12-15 \%$ of early onset dementia. While also characterized by neuronal loss, FTD is histopathologically distinct from AD due to the presence of tau-positive or ubiquitin-positive inclusions. The three main variants are the behavioral or frontal variant (bvFTD; 40\%), progressive non-fluent aphasia (PNFA; 20\%), and semantic dementia (SD; 40\%). The latter two are clinically characterized by a predominance of language impairment and considered subtypes of primary progressive aphasia, which is discussed separately below (Fig. 11.9).

Brain imaging in bv-FTD shows frontal and temporal cortical atrophy with an anterior to posterior gradient, which is bilateral and may be asymmetrical. There may be associated white matter hyperintensities on T2-weighted/T2-FLAIR imaging. Atrophy of the frontal lobe, especially if there is concurrent atrophy of the caudate head, results in disproportionate widening of the frontal horns of the lateral ventricles. In the early stages widening of the sulci in the orbitofrontal region may be seen as an early manifestation of orbital frontal atrophy. Compared with AD, medial temporal lobe atrophy in FTD is more pronounced anteriorly and more asymmetrical.

\subsubsection{Primary Progressive Aphasia}

PPA is a slowly progressive impairment of language, with three subtypes: logopenic progressive aphasia (LPA) (commonly AD pathology, see Sect. 11.5.2), PNFA, and SD (the latter two mainly FTD pathology).

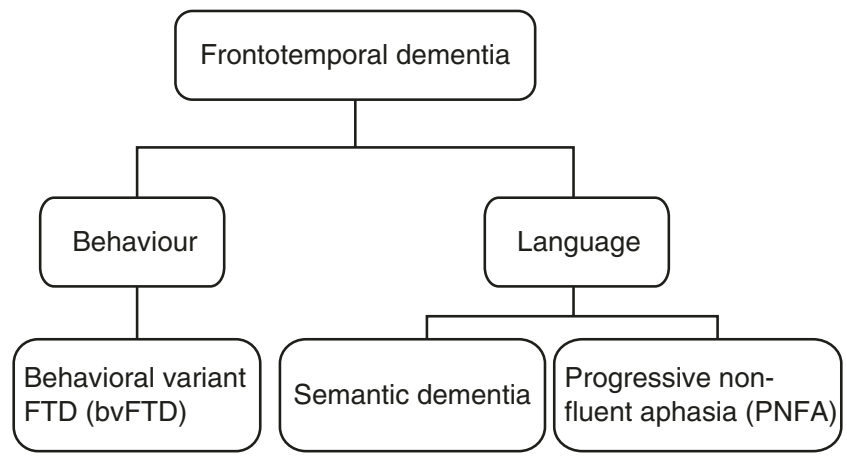

Fig. 11.9 Clinical subtypes of frontotemporal dementia

Patients with PNFA have slow, effortful speech with errors in the production of speech sounds and grammatical deficits. On brain imaging, atrophy is seen in the anterior perisylvian region, involving the inferior, opercular, and insular regions, with a left-hemispheric predominance.

SD is clinically characterized by impairment of semantic performance. Brain imaging shows atrophy of the ventral and lateral regions of the anterior temporal lobes, including the anterior hippocampus and the amygdala. Again, atrophy is often asymmetrical with the left hemisphere more affected than the right, and there is an anterior to posterior gradient.

\subsubsection{Vascular Dementia}

In the elderly, cerebrovascular disease is the second most common pathology underlying dementia after $\mathrm{AD}$. VaD is a heterogeneous entity, including both large and small vessel disease, and involving the gray and/or white matter. By far the most common underlying pathology is small vessel disease, leading to diffuse confluent white matter changes and multiple lacunar infarcts of the deep white and (notably the thalamic) gray matter. Typically, the subcortical U-fibers are spared. When infarction of the deep perforating arteries is complete, lacunar infarcts occur, which are visualized on MRI as small lesions with signal intensity of CSF on all sequences, surrounded by a rim of T2-weighted hyperintensity. A multilacunar state is also known as "état lacunaire," not to be confused with "état criblé," which is the term used for multiple enlarged perivascular spaces in the basal ganglia. Both states are considered pathological and manifestations of small vessel disease. Large vessel disease manifests as multiple (strategic infarcts.

Since cerebrovascular changes are common in healthy elderly, as outlined earlier in this chapter, there are strict criteria to diagnose $\mathrm{VaD}$ set out by the National Institute of Neurological Disorders and Stroke and Association Internationale pour la Recherché et l'Enseignement en Neurosciences (NINDS-AIREN) [19]. A patient must meet the criteria of a dementia syndrome, have evidence of cere- 
brovascular disease on clinical examination and on imaging, and there has to be a temporal relationship between the onset of dementia and cerebrovascular disease.

Radiological criteria are crucial to diagnosis and specified in detail:

- Confluent white matter lesions need to involve at least $25 \%$ of the total white matter to reach the diagnosis of $\mathrm{VaD}$.

- Lacunar infarcts need to involve multiple basal ganglia and the frontal white matter, and thalamic lesions need to be bilateral.

- Strategic_large vessel-infarcts meet the criteria when they involve the following territories: bilateral anterior cerebral artery, paramedian thalamic, inferior medial temporal lobe, parieto-temporal and temporo-occipital association areas and angular gyrus, superior frontal and parietal watershed areas in the dominant hemisphere.

There seems to be a complex interrelationship between AD and cerebrovascular disease that extends beyond the coexistence of these two disease processes. Cerebrovascular disease and $\mathrm{AD}$ often coexist, while stroke often exacerbates pre-existing, sometimes previously subclinical, pathology. Furthermore, $\mathrm{AD}$ and $\mathrm{VaD}$ share common risk factors, such as diabetes and hypertension, as well as genetic factors for brain tissue vulnerability (presenilins, amyloid precursor protein, APOE genes). Microbleeds, considered to be a manifestation of cerebral amyloid angiopathy (CAA), are seen at high frequency in patients with $\mathrm{AD}$. CAA is a microangiopathy with $\beta$-amyloid deposition in the vessel walls. On brain imaging, the presence of two or more microbleeds in a lobar distribution is suggestive of CAA, while other features include subpial siderosis and evidence of past lobar hemorrhage. Although sporadic CAA is commonly seen in the elderly, being the leading cause of lobar hemorrhage, in its severe form it is also recognized as a risk factor for dementia. This typically constitutes a subcortical vascular dementia, but severe CAA is also considered a feature of $\mathrm{AD}$, sharing the APOE $\varepsilon 4$ allele as a common risk factor.

\subsubsection{Dementia with Lewy Bodies (DLB)}

DLB is clinically distinguished from AD by the presence of visually hallucinations, which occur in $80 \%$ of cases. Visual hallucinations may, however, also occur in AD. On brain MRI, there is medial temporal lobe and global cortical atrophy with relative sparing of the primary motor and sensory cortex, as is seen in AD. Atrophy, however, is less pronounced than in $\mathrm{AD}$ when the severity of disease is taken into account. In the early stages of the disease, medial temporal lobe volume may be preserved. Similar to AD, hyperintensity of the white matter on T2-weighted images is seen at higher frequency in DLB than in healthy controls.

\subsubsection{Creutzfeldt-Jakob Disease}

Sporadic CJD, accounting for $85-90 \%$ of cases, generally presents as a subacute dementia syndrome between the ages of 50 and 70 years. Rapid progression and concomitant focal neurological deficits and myoclonus will clinically distinguish CJD from AD during the course of the disease, but initially cognitive symptoms may be similar. Brain MRI typically shows marked hyperintensity on T2-weighted sequences bilaterally in the caudate nucleus and the putamen, and to a lesser extent in the thalamus and neocortex. Diffusion weighted imaging, showing restricted diffusion in the affected gray matter, seems to be the most sensitive sequence to detect CJD-related abnormalities (Table 11.3).

\section{Key Point}

- Structural neuroimaging can help identify underlying causes of dementia through the identification of patterns of atrophy (including symmetry and lobar patterns) as well as presence and distribution of vascular lesions.

Table 11.3 Summary of structural MR imaging findings for the most common dementia subtypes

Typical Alzheimer's disease

- Disproportionate hippocampal atrophy with temporoparietal atrophy

- Relative sparing of the primary motor and sensory cortex

- Bilateral, more or less symmetrical

- Microbleeds in a lobar, subcortical distribution

Atypical Alzheimer's disease

- No or little hippocampal atrophy

- Focal cortical atrophy

- Precuneus: early onset AD

- Parieto-occipital and posterior temporal lobe: PCA

- Posterior temporal cortex and the inferior parietal lobule: LPA

Frontotemporal dementia

- Bilateral frontal and temporal atrophy with anterior to posterior gradient

- Left more than right anterior perisylvian region: PNFA

- Left more than right ventrolateral temporal region: SD

- (Asymmetrical) hippocampal atrophy, more pronounced anteriorly

- Disproportionate widening of the frontal horns: Bv-FTD

- Relative sparing of the parietal and occipital lobes

Creutzfeldt-Jakob disease

- T2-hyperintensity and diffusion restriction bilaterally in the caudate nucleus and putamen, and to a lesser extent in the thalamus and neocortex

Dementia with Lewy bodies

- Medial temporal lobe and global cortical atrophy

- Relative sparing of the primary motor and sensory cortex

- Less extensive than $\mathrm{AD}$ when accounting for disease severity

From Vernooij and Smits [16]; reprinted with permission 


\subsection{Other Dementia Causes}

\subsubsection{Normal Pressure Hydrocephalus (NPH)}

NPH is still a poorly understood disease which seems to result from a disbalance in CSF production and resorption, though an alternative hypothesis is that periventricular white matter damage is a causative factor. The clinical picture is typically characterized by a triad of gait apraxia, dementia, and urinary incontinence; yet, this full trias is only present in less than $33 \%$ of patients. NPH is uncommon under the age of 60 years, and increases in frequency thereafter. As the name suggests, CSF pressure on lumbar puncture is in the normal range. Regardless of underlying etiology, in a number of patients with NPH the clinical symptoms improve with CSF shunting, making this a potentially treatable cause of dementia.

The imaging diagnosis of NPH is challenging. Most striking is the widening of the central CSF spaces (in particular the lateral and third ventricle), with a marked discrepancy between ventricular width and sulcal width. In other words, the ventricular widening cannot be explained by the amount of brain atrophy. A disproportionate widening of the Sylvian fissure can be seen in comparison to the other sulci which appear rather small (Fig. 11.10). Also, some sulci may focally dilate over the convexity or medial surface ("trans-

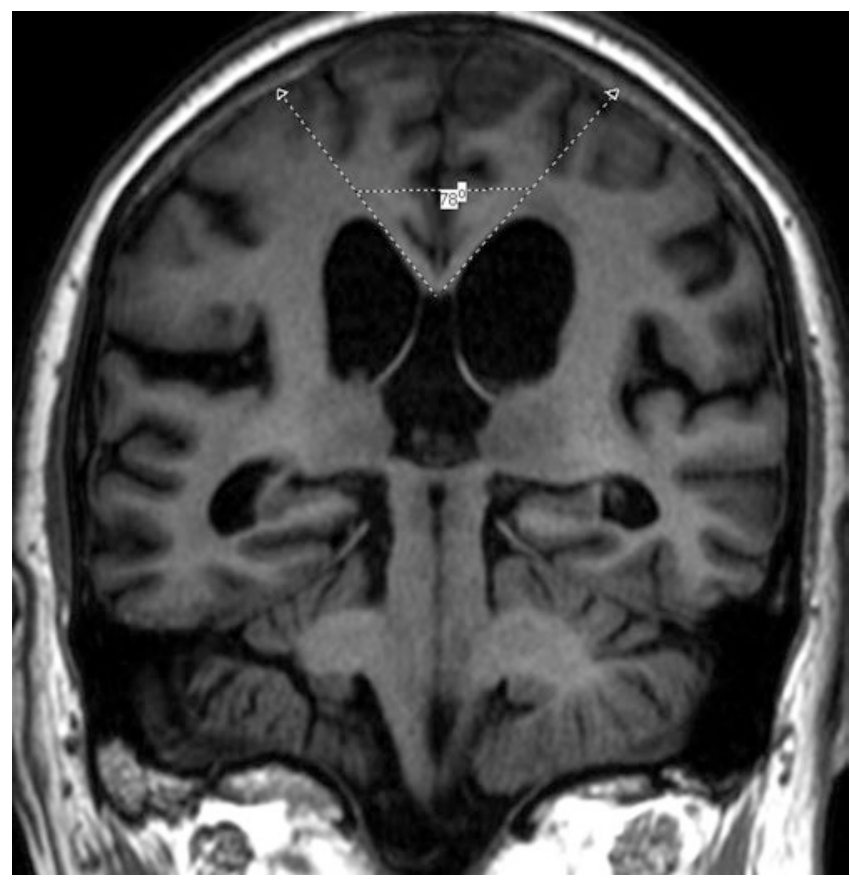

Fig. 11.10 Normal pressure hydrocephalus. Seventy-year-old male with cognitive problems and gait imbalance. MRI shows widening of central CSF spaces, a disproportionate widening of Sylvian fissures relative to other sulci, and crowding of gyri at the vertex/parafalcine region. Corpus callosum angle, measured at the level of the posterior commissure, is $78^{\circ}\left(\right.$ normal $\left.>100^{\circ}\right)$ port sulci"). As the ventricles enlarge, shearing forces are exerted on the white matter, causing in particular the medial lying fibers of the corticospinal tract to be damaged, hence leading to the gait disturbance. This is on imaging exemplified by sharpening of the callosal angle (normal 100-120 $0^{\circ}$, in NPH $50-80^{\circ}$ ) and upward bowing of the corpus callosum, leading to tight high convexity sulci at the vertex and the parafalcine region.

\subsubsection{Corticobasal Syndrome (CBS)}

Corticobasal syndrome is characterized by asymmetric involuntary movements (rigidity, tremor, myoclonus) and apraxia and alien limb phenomena. Additionally, many patients show cognitive deficits or dementia. CBS has several distinct histopathologies, including corticobasal degeneration (tauopathy, related to FTD), and Alzheimer's disease.

Progressive supranuclear palsy, and DLB. While there are no specific imaging findings, CBS with underlying AD pathology usually shows posterior temporal and inferior parietal atrophy, whereas focal atrophy in the premotor cortex or supplemental motor area (often asymmetric) may suggest CBD [20].

\subsubsection{Overlap Between Dementia and Movement Disorders}

There is an overlap between dementia and movement disorders. Parkinson's disease, as the most common neurodegenerative movement disorder, has a cumulative prevalence of dementia of 75-90\% (PD-dementia or PDD). Cognitive dysfunction or dementia is also common in several other movement disorders such as atypical parkinsonism: PSP, DLB, and CBS (see Sect. 11.6.2). Among hyperkinetic disorders, Huntington's disease, spinocerebellar ataxia, and Wilson's disease are best known for their cognitive correlates. Imaging findings are usually dominated by the typical pattern of imaging findings associated with the movement disorder. In some patients cognitive decline may be the dominant clinical manifestation, notably during early stages of the disease, putting a larger emphasis on neuroimaging to aid in the clinical diagnosis.

\subsection{Recommended MR Imaging Protocol in Patients Suspected for Dementia}

The description of imaging findings for the various subtypes above means that the first aims in the imaging evaluation of a patient suspected for dementia should be: 
Table 11.4 Recommended MR imaging protocol for dementia

\begin{tabular}{|c|c|c|}
\hline & Sequence & Findings \\
\hline $\begin{array}{l}\text { 3D } \\
\text { (isotropic) }\end{array}$ & T1-weighted & $\begin{array}{l}\text { Assessment of atrophy (GCA, } \\
\text { MTA) } \\
\text { Coronal plane: hippocampal } \\
\text { atrophy. } \\
\text { Sagittal plane: precuneus atrophy }\end{array}$ \\
\hline $2 \mathrm{D} / 3 \mathrm{D}$ & T2-FLAIR & $\begin{array}{l}\text { Atrophy, white and gray matter } \\
\text { signal abnormalities } \\
\text { Coronal plane: hippocampal signal } \\
\text { abnormalities } \\
\text { WMH and lacunes }\end{array}$ \\
\hline $\begin{array}{l}2 \mathrm{D} \\
\text { transverse }\end{array}$ & $\begin{array}{l}\text { TSE/FSE } \\
\text { T2-weighted }\end{array}$ & $\begin{array}{l}\text { Lesions in thalamus and posterior } \\
\text { fossa }\end{array}$ \\
\hline $\begin{array}{l}2 \mathrm{D} \\
\text { transverse }\end{array}$ & DWI & $\begin{array}{l}\text { Diffusion restriction: recent } \\
\text { ischemia; CJD }\end{array}$ \\
\hline $\begin{array}{l}2 \mathrm{D} \\
\text { transverse }\end{array}$ & T2*-weighted & Microbleeds, superficial siderosis \\
\hline
\end{tabular}

(a) To assess the extent and pattern of brain atrophy, in particular lobar cortical atrophy (with GCA) and medial temporal lobe atrophy (MTA; for evidence of Alzheimer's pathology);

(b) To determine the degree of vascular damage, including the occurrence of strategic vascular lesions.

We recommend that the MRI scanning protocol includes T1-weighted, T2-weighted, T2-FLAIR, diffusion weighted, and T2*-weighted sequences (Table 11.4). Routine application of contrast is not indicated, although it may be needed in atypical cases when granulomatous disease, vasculitis, and infection are considered as differential diagnoses. 3D T1-weighted images should be reconstructed in a coronal plane perpendicular to the long axis of the hippocampus for adequate assessment of medial temporal atrophy.

\subsection{Reporting Findings in Dementia}

Reporting MRI findings in dementia includes both those pointing towards the pathology underlying the dementia syndrome and those suggesting an alternative cause of neurocognitive degeneration. The latter would include focal abnormalities such as brain tumor or subdural hematoma. The hippocampus needs to be scrutinized for signal abnormality on T2-weighted sequences to diagnose ischemia or sclerosis. Areas of diffusion restriction may indicate pathologies such as acute ischemia, herpes simplex encephalitis, alcoholic encephalopathy, or CJD. T2-weighted hyperintensity in certain brain regions may point to a specific diagnosis, such as in the periaqueductal gray or mammillary bodies in case of alcoholic encephalopathy.

The radiological report further needs to include a structured and standardized assessment of the global cortical atrophy, the medial temporal lobe and white matter lesion load, according to, for instance, the GCA, MTA, and Fazekas visual rating scales, respectively (see Tables 11.1 and 11.2, Figs. 11.3, 11.4, and 11.5). Atrophy should always be rated on the same imaging sequence, preferably the FLAIR or T1-weighted sequence. The degree of asymmetry, as well as focal regions of atrophy should be reported separately. Both the number and distribution of microbleeds need to be reported. Large and small vessel cerebrovascular changes need to be specified according to the topographic and severity operational NINDS-AIREN criteria to enable diagnosis of $\mathrm{VaD}$.

\subsection{Concluding Remarks}

The dementia syndrome encompasses a wide variety of underlying causal pathologies, for many of which neuroimaging can give additional information in the diagnostic workup. It is important for (neuro)radiologists to read imaging exams from memory clinic patients against a background of normal aging brain pathology. Patterns of atrophy and vascular lesions can direct towards specific underlying diagnoses. Standardized image acquisition and structured reporting (including the use of semi-quantitative visual rating scales) will increase diagnostic yield and facilitate communication with referring clinicians.

\section{Take Home Messages}

- Dementia is a complex clinical syndrome with multiple underlying causes, of which presentation and prevalence differ according to patient demographics.

- Knowledge on typical and less typical imaging presentations of the various causes of dementia is essential in order to exploit the value of neuroimaging in the memory clinic to its full potential.

- Radiologists should perform a standardized imaging protocol, which enables both the assessment of patterns of atrophy and vascular lesions, and employ a systematic assessment (using semi-quantitative visual rating scales) in order to improve diagnostic yield.

\section{References}

1. Sachdev PS, Blacker D, Blazer DG, Ganguli M, Jeste DV, Paulsen JS, et al. Classifying neurocognitive disorders: the DSM-5 approach. Nat Rev Neurol. 2014;10(11):634-42.

2. Rizzi L, Rosset I, Roriz-Cruz M. Global epidemiology of dementia: Alzheimer's and vascular types. Biomed Res Int. 2014;2014:908915.

3. Lobo A, Launer LJ, Fratiglioni L, Andersen K, Di Carlo A, Breteler MM, et al. Prevalence of dementia and major subtypes in Europe: 
a collaborative study of population-based cohorts. Neurologic Diseases in the Elderly Research Group. Neurology. 2000;54(11 Suppl 5):S4-9.

4. Goodman RA, Lochner KA, Thambisetty M, Wingo TS, Posner SF, Ling SM. Prevalence of dementia subtypes in United States Medicare fee-for-service beneficiaries, 2011-2013. Alzheimer's Dement. 2017;13(1):28-37.

5. van der Flier WM, Scheltens P. Epidemiology and risk factors of dementia. J Neurol Neurosurg Psychiatry. 2005;76(Suppl 5):v2-7.

6. McMurtray A, Clark DG, Christine D, Mendez MF. Early-onset dementia: frequency and causes compared to late-onset dementia. Dement Geriatr Cogn Disord. 2006;21(2):59-64.

7. Picard C, Pasquier F, Martinaud O, Hannequin D, Godefroy O. Early onset dementia: characteristics in a large cohort from academic memory clinics. Alzheimer Dis Assoc Disord. 2011; 25(3):203-5.

8. Schneider JA, Arvanitakis Z, Bang W, Bennett DA. Mixed brain pathologies account for most dementia cases in communitydwelling older persons. Neurology. 2007;69(24):2197-204.

9. Brunnstrom H, Gustafson L, Passant U, Englund E. Prevalence of dementia subtypes: a 30-year retrospective survey of neuropathological reports. Arch Gerontol Geriatr. 2009;49(1):146-9.

10. Jack CR Jr, Bennett DA, Blennow K, Carrillo MC, Feldman HH, Frisoni GB, et al. A/T/N: an unbiased descriptive classification scheme for Alzheimer disease biomarkers. Neurology. 2016;87(5):539-47.

11. McKhann GM, Knopman DS, Chertkow H, Hyman BT, Jack $\mathrm{CR}$ Jr, Kawas $\mathrm{CH}$, et al. The diagnosis of dementia due to Alzheimer's disease: recommendations from the National Institute on Aging-Alzheimer's Association workgroups on diagnostic guidelines for Alzheimer's disease. Alzheimer's Dement. 2011;7(3):263-9.
12. Vernooij MW, Barkhof F. Neuroimaging in normal brain aging. In: Barkhof F, Jäger H, Thurnher M, Rovira À, editors. Clinical neuroradiology. Cham: Springer; 2019.

13. Ikram MA, Vrooman HA, Vernooij MW, van der Lijn F, Hofman $A$, van der Lugt A, et al. Brain tissue volumes in the general elderly population. The Rotterdam Scan Study. Neurobiol Aging. 2008;29(6):882-90.

14. Pasquier F, Leys D, Weerts JG, Mounier-Vehier F, Barkhof F, Scheltens P. Inter- and intraobserver reproducibility of cerebral atrophy assessment on MRI scans with hemispheric infarcts. Eur Neurol. 1996;36(5):268-72.

15. Scheltens P, Leys D, Barkhof F, Huglo D, Weinstein HC, Vermersch $\mathrm{P}$, et al. Atrophy of medial temporal lobes on MRI in "probable" Alzheimer's disease and normal ageing: diagnostic value and neuropsychological correlates. J Neurol Neurosurg Psychiatry. 1992;55(10):967-72.

16. Vernooij MW, Smits M. Structural neuroimaging in aging and Alzheimer's disease. Neuroimaging Clin N Am. 2012;22(1):33-55, vii-viii.

17. Pereira JB, Cavallin L, Spulber G, Aguilar C, Mecocci P, Vellas B, et al. Influence of age, disease onset and ApoE4 on visual medial temporal lobe atrophy cut-offs. J Intern Med. 2014;275(3):317-30.

18. Vermeer SE, Longstreth WT Jr, Koudstaal PJ. Silent brain infarcts: a systematic review. Lancet Neurol. 2007;6(7):611-9.

19. Roman GC, Tatemichi TK, Erkinjuntti T, Cummings JL, Masdeu $\mathrm{JC}$, Garcia JH, et al. Vascular dementia: diagnostic criteria for research studies. Report of the NINDS-AIREN International Workshop. Neurology. 1993;43(2):250-60.

20. Chahine LM, Rebeiz T, Rebeiz JJ, Grossman M, Gross RG. Corticobasal syndrome: five new things. Neurol Clin Pract. 2014;4(4):304-12.

Open Access This chapter is licensed under the terms of the Creative Commons Attribution 4.0 International License (http://creativecommons. org/licenses/by/4.0/), which permits use, sharing, adaptation, distribution and reproduction in any medium or format, as long as you give appropriate credit to the original author(s) and the source, provide a link to the Creative Commons license and indicate if changes were made.

The images or other third party material in this chapter are included in the chapter's Creative Commons license, unless indicated otherwise in a credit line to the material. If material is not included in the chapter's Creative Commons license and your intended use is not permitted by statutory regulation or exceeds the permitted use, you will need to obtain permission directly from the copyright holder. 